\title{
A FAMILY OF EXPONENTIALLY FITTED MULTIDERIVATIVE METHOD FOR STIFF DIFFERENTIAL EQUATIONS
}

\author{
ABHULIMENC.E* AND UKPEBOR L.A \\ Department Of Mathematics, \\ Ambrose Alli University, Ekpoma, Nigeria.
}

\begin{abstract}
.
In this paper, an A-stable exponentially fitted predictor-corrector using multiderivative linear multistep method for solving stiff differential equations is developed. The method which is a two-step third derivative method of order five contains free parameters. The numerical stability analysis of the method was discussed, and found to be A-stable. Numerical examples are provided to show the efficiency of the method when compared with existing methods in the literature that have solved the set of problems.
\end{abstract}

Keywords: Exponentially fitted, Multiderivative linear multistep, stiff differential equations, A-stable.

\subsection{INTRODUCTION}

In order to solve stiff initial value problems in ordinary differential equations efficiently, many new methods have been developed in recent years, which satisfy certain stability requirements.

The property of A-stability is desirable in formulas to be used in the solution of stiff systems of differential equations especially from chemical kinetics and the discretisation of partial differential equations. Dahlquist [7], proved that A-stable linear multistep formulas must be implicit, its maximum order is two and of those of second order, the one with the smallest truncation error coefficient is the trapezoidal rule. In nearly every linear systems of differential equation, which have widely disperse eignevalues, high order A-stable formulas are particularly appropriate since they allow integration to proceed with a larger step size.

Thus, the need to develop a high order A-stable implicit multistep formula, which uses linear combinations of derivatives higher than the first, give rise to the development of multiderivative multistep formulas. Lambert [11], and Enright [8], pointed out that Multiderivative methods give high accuracy and possess good stability properties when used to solve first order initial value problems in ordinary differential equation.

However, A-stable multiderivative multistep formulas designed for solving stiff systems of differential equations includes, Enright [8], Jackson and Kenue[10], Brown [5],Okunuga[13], Otunta and Abhulimen[15],[3]Abhulimen and Omeike[2].

\subsection{THE GENERAL MULTIDERIVATIVE MULTISTEP METHOD}

The general multiderivative multistep method is given by,

$$
\sum_{i=0}^{k} \alpha_{i} y_{n+i}=\sum_{j=1}^{s} h^{j} \sum_{i=0}^{k} \gamma_{j, i} f_{n+i}^{(j-1)}, \quad n=0,1,2
$$

Where $f_{n+i}^{(j)}$ is the $j^{\text {th }}$ derivative of $f(x, y)$ evaluated at $\left(x_{n+1}, y_{n+1}\right), \alpha_{i}$ and $\gamma_{j, i}$ are real constants with $\alpha_{k} \neq 0$ and $y_{n+1}$ is the appropriate numerical solution evaluated at the point $x_{n+i}$. In order to remove the arbitrary constant in (2.1) we shall always assume that $\alpha_{k}=+1$, and $\sum_{i=0}^{k}\left|\alpha_{i}\right|>0$ and $\sum_{i=0}^{k}\left|\gamma_{j, i}\right|>0 j=1,2, \ldots, s$

\subsection{DEVELOPMENT OF NEW EXPONENTIALLY FITTED MULTIDERIVATIVE METHODS}

A numerical integration formula is said to be exponentially fitted at (complex) value $\lambda=\lambda_{0}$ if when the method is applied to the scalar test problem $y^{\prime}=\lambda h, y\left(x_{0}\right)=y_{0}$, with exact initial condition, the characteristic equation $\phi(\lambda h)$ satisfies the relation $\phi\left(\lambda_{0} h\right)=e^{\lambda_{0} h}$.

However, the idea of using exponentially fitted formulas for the appropriate numerical integration of certain classes of stiff systems of first order ordinary differential equation of the form,

$$
y^{\prime}=f(x, y), \quad y\left(x_{0}\right)=y_{0}
$$

which was originally proposed by Liniger and Willoughby [12], is to derive integration formulas containing free parameters (other than the step length of integration) and then to choose these parameters so that a given function ( $e^{q}$ ), where $q$ is real, satisfies the integration formula exactly.

Liniger and Willoughby [12] derived three 1-step integration formulas with orders ranging from $1-3$. Their results revealed that for all choices of the fitting parameter $q$, their formulas are A-stable. 


\subsection{DERIVATION OF FAMILY OF TWO STEP EXPONENTIALLY FITTED METHODS}

The objective of this paper is to develop a two-step, third derivative multiderivative exponentially fitted formulas. (i.e. $k=2$ and $s=3$ ).

For this purpose, equation (2.1) is reduced to,

$$
\sum_{i=0}^{k} \alpha_{i} y_{n+i}=h \sum_{i=0}^{k} \gamma_{1, i} f_{n+i}+h^{2} \sum_{i=0}^{k} \gamma_{2, i} f_{n+i}^{(1)}+h^{3} \sum_{i=0}^{k} \gamma_{3, i} f_{n+i}^{(2)}
$$

Where $\beta_{i}=\gamma_{1, i}, \phi=\gamma_{2, i}, \omega_{i}=\gamma_{3, i}$

From equation (2.2), we obtain

$$
\sum_{i=0}^{k} \alpha_{i} y_{n+i}=h \sum_{i=0}^{k} \beta_{i} f_{n+i}+h^{2} \sum_{i=0}^{k} \phi_{i} g_{n+i}+h^{3} \sum_{i=0}^{k} \omega_{i} v_{n+i}
$$

And

$$
\sum_{i=0}^{k} \alpha_{i} y_{n+i}=h \sum_{i=0}^{k+1} \beta_{i} f_{n+i}+h^{2} \sum_{i=0}^{k} \phi_{i} g_{n+i}+h^{3} \sum_{i=0}^{k} \omega_{i} v_{n+i}
$$

However, the implementation of the proposed formulas involves a pair of formulas, that is predictor and corrector formulas. Thus, equation (2.3) serves as the predictor and while equation (2.4) serves as the corrector.

where,

$f_{n+i}=f\left[x_{n+i}, y\left(x_{n+i}\right)\right]=y_{n+i}^{\prime}$ and $g_{n+i}=f^{\prime}\left[x_{n+i}, y\left(x_{n+i}\right)\right]=y_{n+i}^{\prime \prime}$

$v_{n+i}=f^{\prime \prime}\left[x_{n+i}, y\left(x_{n+i}\right)\right]=y_{n+i}^{\prime \prime \prime}$

are respectively the first, second and third derivatives of $y_{n+i}$. When we are deriving exponentially fitted multistep methods, the approach is to allow both (2.3) and (2.4) to posses free parameters order than the mesh size ' $h$ ' which allow it to be fitted automatically to exponential function.

\subsection{DERIVATION OF METHOD OF FIFTH ORDER FORMULAS}

The derivation of predictor-corrector integrations formula of order 5 involves two stages as was done in higher order. First we derived the order four predictor by setting $c_{0}=c_{1}=c_{2}=c_{3}=c_{4}=0$ in equation (2.3) to obtain five set of equations with 12 unknown parameters and we then obtain the following set of simultaneous equations

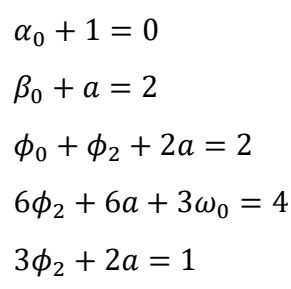<smiles>CCCCCC</smiles>

When we solve the above equations we obtain,

$$
\bar{\alpha}_{0}=-1, \bar{\alpha}_{2}=1, \bar{\beta}_{0}=2-a, \bar{\phi}_{0}=\frac{5}{3}-\frac{4}{3} a, \bar{\phi}_{2}=\frac{1}{3}-\frac{2}{3} a, \bar{\omega}_{0}=\frac{2}{3}-\frac{2}{3} a
$$

When these values of the parameters are substituted into (2.3), we obtain the predictor formula as:

$$
\bar{y}_{n+2}-\bar{y}_{n}=h\left[a y_{n+2}^{\prime}+(2-a) y_{n}^{\prime}\right]+h^{2}\left[\left(\frac{1}{3}-\frac{2}{3} a\right) y_{n+2}^{\prime \prime}+\left(\frac{5}{3}-\frac{4}{3} a\right) y_{n}^{\prime \prime}\right]+h^{3}\left[\left(\frac{2}{3}-\frac{2}{3} a\right) y_{n}^{\prime \prime \prime}\right]
$$

Now, for exponential fitting purpose, we apply (2.7) to scalar test function

$$
y^{\prime}=\lambda y, \quad y(0)=1
$$

to obtain equation (2.8) below.

$$
\frac{\bar{y}_{n+2}}{y_{n}}=\frac{1+(2-a) q+\left(\frac{5}{3}-\frac{4}{3} a\right) q^{2}+\left(\frac{2}{3}-\frac{2}{3} a\right) q^{3}}{1+a q-\left(\frac{1}{3}-\frac{2}{3} a\right) q^{2}}=R(\bar{q})
$$


For the purpose of stability analysis, we obtain the free parameter $a$ from (2.9).

But from equation ... $\frac{\bar{y}_{n+2}}{y_{n}}=e^{2 q}$ then equation (2.9) yields,

$$
a=\frac{1+2 q+\frac{1}{3} q^{2}\left(5+e^{2 q}\right)+\frac{2}{3} q^{3}-e^{2 q}}{\frac{2}{3} q^{2}\left(2+e^{2 q}\right)+q\left(1+e^{2 q}\right)+\frac{2}{3} q^{3}}
$$

Again to obtain the corresponding order 5 corrector formula, we obtain six set of simultaneous equation from (2.4)asfollows,

$$
\begin{aligned}
& \alpha_{0}+1=0 \\
& \beta_{0}+b+\beta_{2}=2 \\
& 2 \beta_{2}+3 b+\phi_{0}+\phi_{2}=2 \\
& 6 \beta_{2}+\frac{27}{2} b+6 \phi_{2}+3 \omega_{0}=4 \\
& 4 \beta_{2}+\frac{27}{2} b+6 \phi_{2}=2 \\
& 10 \beta_{2}+\frac{405}{8} b+20 \phi_{2}=4
\end{aligned}
$$

We impose the same condition as in predictor, and in addition, we let $\beta_{3}=b$ as free parameters $\alpha_{2}=1$, the values of the unknown parameters are obtained from (2.11) as,

$$
\begin{gathered}
\alpha_{0}=-1, \alpha_{2}=1, \beta_{0}=\frac{6}{5}-\frac{43}{16} b, \beta_{2}=\frac{4}{5}+\frac{27}{16} b, \beta_{3}=b \\
\phi_{0}=\frac{3}{5}-3 b, \phi_{2}=-\frac{1}{5}-\frac{27}{8} b, \omega_{0}=\frac{2}{15}-\frac{9}{8} b
\end{gathered}
$$

When these values are substitute into (2.4), we obtain the fifth order corrector formula as,

$y_{n+2}-y_{n}=h\left[b_{3} y_{n+3}^{\prime}+\left(\frac{4}{5}+\frac{27}{16} b\right) y_{n+2}^{\prime}+\left(\frac{6}{5}-\frac{43}{16} b\right) y_{n}^{\prime}\right]+h^{2}\left[\left(-\frac{1}{5}-\frac{27}{8} b\right) y_{n+2}^{\prime \prime}+\left(\frac{8}{5}+\frac{33}{16} b\right) y_{n}^{\prime \prime}\right]+h^{3}\left[\left(\frac{2}{15}-\frac{9}{8} b\right) y_{n}^{\prime \prime \prime}\right]$

By applying (2.12) to test function (2.8) we obtain,

$$
\frac{y_{n+2}}{y_{n}}=\frac{1+\left(\frac{6}{5}-\frac{43}{16} b\right) q+b q \cdot \frac{y_{n+3}}{y_{n}}+\left(\frac{8}{5}+\frac{33}{16} b\right) q^{2}+\left(\frac{2}{15}-\frac{9}{8} b\right) q^{3}}{1-\left(\frac{4}{5}+\frac{27}{16} b\right) q+\left(\frac{1}{5}+\frac{27}{8} b\right) q^{2}}=R(q)
$$

We need to obtain the relation $\frac{y_{n+3}}{y_{n}}$ in (2.13) for the purpose of exponential fitting condition and stability. From the solution of test function (2.8), we established that;

$$
\begin{aligned}
& \qquad \frac{y_{n+2}}{y_{n}}=e^{2 q}=R(\bar{q}) \\
& \text { Also, } \frac{y_{n+3}}{y_{n}}=e^{3 q}=\left(e^{2 q}\right)^{\frac{3}{2}} \\
& \qquad \frac{\bar{y}_{n+3}}{y_{n}}=[R(\bar{q})]^{\frac{3}{2}}
\end{aligned}
$$

Equation (2.13) now becomes

$\frac{y_{n+2}}{y_{n}}=\frac{1+\left(\frac{6}{5}-\frac{43}{16} b\right) q+b q \cdot\left[\bar{R}_{2}(q)\right]^{\frac{3}{2}}+\left(\frac{3}{5}-3 b\right) q^{2}+\left(\frac{2}{15}-\frac{9}{8} b\right) q^{3}}{1-\left(\frac{4}{5}+\frac{27}{16} b\right) q+\left(\frac{1}{5}+\frac{27}{8} b\right) q^{2}}=R(q)$

Equation (2.15) now unites both the parameter and corrector formulas which is capable of solving stiff systems. We obtain the value of the free parameter $b$ from (2.15) as, 


$$
b(q)=\frac{1+\frac{1}{5}\left(b q+3 q^{2}\right)+\frac{2}{15} q^{3}+\frac{4}{5} q e^{2 q}-e^{2 q}\left(1+\frac{1}{5} q^{2}\right)}{\frac{27}{8} q^{2} e^{2 q}-3 q^{2}+\frac{9}{8} q^{3}-\frac{1}{16}\left(27 q e^{2 q}-43 q\right)-q e^{3 q}}
$$

\subsection{STABILITY CONSDIERATION OF THE METHOD}

To examine the stability conditions required by this method, it is expected by maximum modulus theorem that the stability function of the method given by (2.16) satisfies $|R(q)|<1$.

In order to determine the interval of absolute stability of the method, we find limits of both $a(q)$ and $b(q)$ as $q \rightarrow 0$ and $q \rightarrow-\infty$

$\operatorname{Lim}_{q \rightarrow-\infty} a(q)=\operatorname{Lim}_{q \rightarrow-\infty}\left[\frac{1+2 q+\frac{1}{3} q^{2}\left(5+e^{2 q}\right)+\frac{2}{3} q^{3}-e^{2 q}}{\frac{2}{3} q^{2}\left(2+e^{2 q}\right)+q\left(1+e^{2 q}\right)+\frac{2}{3} q^{3}}\right]=1$

$\operatorname{Lim}_{q \rightarrow 0} a(q)=\operatorname{Lim}_{q \rightarrow 0}\left[\frac{1+2 q+\frac{1}{3} q^{2}\left(5+e^{2 q}\right)+\frac{2}{3} q^{3}-e^{2 q}}{\frac{2}{3} q^{2}\left(2+e^{2 q}\right)+q\left(1+e^{2 q}\right)+\frac{2}{3} q^{3}}\right]=\frac{4}{5}$

That is $a \in\left(\frac{4}{5}, 1\right)$ of $q \in(-\infty, 0]$,

Similarly, from (2.16) we have,

$\operatorname{Lim}_{q \rightarrow-\infty} b(q)=\operatorname{Lim}_{q \rightarrow-\infty}\left[\frac{1+\frac{1}{5}\left(6 q-8 q^{2}\right)+\frac{2}{15} q^{3}+\frac{4}{5} q e^{2 q}-e^{2 q}\left(1+\frac{1}{5} q^{2}\right)}{\frac{27}{8} q^{2} e^{2 q}-\frac{33}{16} q^{2}+\frac{9}{8} q^{3}-\frac{1}{16}\left(27 q e^{2 q}-43 q\right)-q e^{3 q}}\right]=\frac{16}{135}$

$\operatorname{Lim}_{q \rightarrow 0} b(q)=\operatorname{Lim}_{q \rightarrow 0}\left[\frac{1+\frac{1}{5}\left(6 q-8 q^{2}\right)+\frac{2}{15} q^{3}+\frac{4}{5} q e^{2 q}-e^{2 q}\left(1+\frac{1}{5} q^{2}\right)}{\frac{27}{8} q^{2} e^{2 q}-\frac{33}{16} q^{2}+\frac{9}{8} q^{3}-\frac{1}{16}\left(27 q e^{2 q}-43 q\right)-q e^{3 q}}\right]=\frac{8}{405}$

Thus we formed that $a \in\left(\frac{4}{5}, 1\right)$ and $b \in\left(\frac{8}{405}, \frac{16}{135}\right)$

Now, we further verify analytically that the ranges of value of $a$ and $b$ represent the region of absolute stability of the new method. We illustrate this by taking a large sample $S$ as shown in table 3.1 below;

Table 3.1: Parameters values of $\mathbf{a}$ and $\mathbf{b}$

\begin{tabular}{|c|c|c|}
\hline $\boldsymbol{q}$ & $\boldsymbol{a}$ & $\boldsymbol{b}$ \\
\hline-10 & 0.955214724 & 0.0989973 \\
\hline-100 & 0.995050247 & 0.1163663 \\
\hline-500 & 0.999002002 & 0.1180848 \\
\hline-1000 & 0.999500500 & 0.1183014 \\
\hline-5000 & 0.999900020 & 0.1184751 \\
\hline-10000 & 0.999950005 & 0.1184968 \\
\hline-20000 & 0.999975001 & 0.11850765 \\
\hline
\end{tabular}

From the table above, we observed that as $q$ decreases, the values of $a$ andbare monotonically increasing.

The implication of the observation is that, for any of value of $q$ in the open left-half plane, the corresponding values of $a(q)$ and $b(q)$ converges and bounded within the ranges of $a \in\left(\frac{4}{5}, 1\right)$ and $b \in\left(\frac{8}{405}, \frac{16}{135}\right)$ respectively. These ranges help to determine the region of absolute stability of the new method.

Furthermore, we examine the stability function of the new method. From equation (2.15), the stability function of the new method is given as 


$$
\tau^{*}=\frac{1+\left(\frac{6}{5}-\frac{43}{16} b\right) q+b q \cdot\left[\bar{R}_{2}(q)\right]^{\frac{3}{2}}+\left(\frac{3}{5}-3 b\right) q^{2}+\left(\frac{2}{15}-\frac{9}{8} b\right) q^{3}}{1-\left(\frac{4}{5}+\frac{27}{16} b\right) q+\left(\frac{1}{5}+\frac{27}{8} b\right) q^{2}}
$$

When we tested for the values of $q \in(-\infty, 0]$, with the region of absolute stability $a \in\left(\frac{4}{5}, 1\right)$ and $b \in\left(\frac{8}{405}, \frac{16}{135}\right)$, we observed that $\left|\tau^{*}(q)\right|<1$. Hence, by definition of A-stability of multistep method, we conclude that the new method is Astable. The new method can solve stiff problems in ordinary differential equations.

\subsection{NUMERICAL EXPERIMENTS}

In the section, the Numerical Integrators derived are tested on several standard stiff value problems (IVPs) in Ordinary Differential Equations (ODEs).

To show the effectiveness and validity of our newly derived methods, we present some numerical examples below. All numerical examples are coded in Fortan 77 and implemented on digital computer.

However, for purpose of comparative analysis on the performance of the new scheme we denote $A L^{5}$ as the new method, $\mathrm{CH} 4$, CH5-Cash [6] method of order 4 and 5 respectively, J-K Jackson and Kenue[10], OK6-Okunuga [13], AB7, AB8, NM9 represent Abhulimen and Otunta[3] method of order 7, 8 and 9 respectively. $\mathrm{F}^{5}$ Abhulimen[4] method of order 5, AF5Abhulimen and Okunuga[1] and AG6-Abhulimen and Omeike[2].

\section{Example 1}

Non-linear stiff problems (Enright and Pryce) [9]

$$
\begin{aligned}
& y_{1}^{\prime}=-0.013 y_{1}+1000 y_{1} y_{3}, \quad y_{1}(0)=1 \\
& y_{2}^{\prime}=-2500 y_{2} y_{3} \text {, } \\
& \left.y_{2}(0)=1\right\} \\
& y_{3}^{\prime}=0.013 y_{1}-1020 y_{1} y_{3}-2500 y_{2} y_{3}, \quad y_{3}(0)=0
\end{aligned}
$$

\begin{tabular}{|c|c|c|c|c|}
\hline Step length $h$ & Method & $y_{1}$ & $y_{2}(1)$ & $y_{3}(1)$ \\
\hline \multirow[t]{2}{*}{0.0625} & $\mathrm{AL}^{5}$ & 0.5882826881 & 1.0092403605 & -2.7914604750 \\
\hline & AB8 & 0.5884667145 & 1.0090563343 & -2.7919757498 \\
\hline \multirow[t]{2}{*}{0.1} & $\mathrm{AL}^{5}$ & 0.5882826902 & 1.0092403584 & -2.7914604809 \\
\hline & AB8 & 0.5882826902 & 1.0092403584 & -2.7914604809 \\
\hline \multicolumn{2}{|l|}{ Exact Solution } & 0.5882826881 & 1.0092403605 & -2.7914604750 \\
\hline \multicolumn{5}{|c|}{ Errors } \\
\hline \multirow[t]{2}{*}{0.0625} & $\mathrm{AL}^{5}$ & $1.5 \times 10^{-6}$ & $1.5 \times 10^{-6}$ & $2.5 \times 10^{-6}$ \\
\hline & AB8 & $-1.8 \times 10^{-4}$ & $1.8 \times 10^{-4}$ & $5.2 \times 10^{-4}$ \\
\hline \multirow[t]{2}{*}{0.1} & $\mathrm{AL}^{5}$ & $1.2 \times 10^{-10}$ & $1.2 \times 10^{-10}$ & $4.2 \times 10^{-10}$ \\
\hline & AB8 & $2.2 \times 10^{-8}$ & $2.2 \times 10^{-9}$ & $6.3 \times 10^{-9}$ \\
\hline
\end{tabular}

Table 4.1 Numerical result of the non-linear stiff problem

\section{Example 2}

Second order differential equation (Okunuga[13])

The accuracy of the new method is also illustrated

$\frac{d^{2} y}{d x^{2}}+1001 \frac{d y}{d x}+100 y=0$

$y(0)=1, y^{\prime}(0)=1$ 
The system (4.2) can be rewritten as a first - order system;

$\frac{d y_{1}}{d x}=y_{2} \quad y_{1}(0)=1$
$\frac{d y_{2}}{d x}=-1001 y_{2}-1000 y_{1} y_{2}(0)=-1$

Thus we obtain a $2 \times 2$ system of stiff IVP. The eigenvalues of the Jacobian matrix

$$
\lambda_{1}=-1000
$$

The general solution of (4.2) is $y(x)=A e^{-x}+B e^{-1000 x}$. If we impose the initial conditions in $0 \leq x \leq 1$ the exact solution is $y(x)=e^{-x}$.

The result of this problem using the newly derived methods are obtained at $x=1$; as given in table 4.2 below.

\section{Table 4.2: Numerical results on second order ODE at $x=1$}

\begin{tabular}{|l|l|c|c|}
\hline Step $\boldsymbol{h}$ & Method & $\boldsymbol{y}(\mathbf{1})$ & Error $(\boldsymbol{y})$ \\
\hline 0.05 & OK6 & 0.367879436 & $5.6 \times 10^{-8}$ \\
\hline & AG6 & 0.36787846 & $1.4 \times 10^{-8}$ \\
\hline & AF5 & 0.36787930 & $1.8 \times 10^{--}$ \\
\hline & AL $^{5}$ & $\mathbf{0 . 3 6 7 8 7 9 4 3}$ & $\mathbf{5 . 2 \times 1 0 ^ { - 9 }}$ \\
\hline $\mathbf{0 . 1 2 5}$ & AL $^{5}$ & $\mathbf{0 . 3 6 7 8 7 9 4 4}$ & $\mathbf{2 . 7 \times 1 0 ^ { - 8 }}$ \\
\hline \multicolumn{2}{|l|}{ Exact Solution } & 0.367879435 & \\
\hline
\end{tabular}

It will be observed from table 4.2 above, for $h=0.05$ the numerical result of the problem consider reveal that the new method perform better with existing methods in the literature. The level of performing of better

\section{Example 3}

Jackson and Kenue[10], Cash [6], Okunuga[4]

$y^{\prime}=-y+95 z ; \quad y(0)=1$

$z^{\prime}=-y-97 z ; \quad y(0)=1$

$x \in[0,1]$

The eigenvalues of the Jacobian matrix of the system are $\lambda_{1}=-2$ and $\lambda_{2}=-96$ with stiffness ratio 48 .

The exact solution is given as,

$$
\begin{gathered}
y=\left(95 e^{-2 x}-48 e^{-96 x}\right) / 47 \\
z=\left(48 e^{-96 x}-e^{-2 x}\right) / 47
\end{gathered}
$$

For comparison purpose, we have the following as;

AB7, AB8 and NM9 to represent Abhulimen and Otunta "Two step third derivative methods order seven, eight and nine" respectively. $\mathrm{F}^{5}$ denote three-step second derivative scheme.

Table 4.3: Comparative analysis of result of problem 3 at $x=1$

\begin{tabular}{|l|l|l|l|}
\hline Method & Step size & $y_{1}($ /error/) & $z(1) \times 10^{2}($ /error/) \\
\hline J-K & $h=0.0625$ & $0.2725503\left(3.0 \times 10^{-1}\right)$ & $-0.2879477\left(4.0 \times 10^{-9}\right)$ \\
\hline CH4 & & $0.2735498\left(3.0 \times 10^{-1}\right)$ & $-0.2879471\left(3.0 \times 10^{-9}\right)$ \\
\hline CH5 & & $0.27355005\left(3.0 \times 10^{-8}\right)$ & $-0.28794742\left(3.0 \times 10^{-9}\right)$ \\
\hline AB7 & & $0.27354004\left(4.0 \times 10^{-5}\right)$ & $-0.28796321\left(6.0 \times 10^{-5}\right)$ \\
\hline$F^{4}$ & & $0.2735503\left(3.0 \times 10^{-1}\right)$ & $-0.2879477\left(3.1 \times 10^{-1}\right)$ \\
\hline NM9 & & $0.27354004\left(7.9 \times 10^{-5}\right)$ & $-0.28794740\left(8.3 \times 10^{-1}\right)$ \\
\hline$F^{5}$ & & $0.27355003\left(6.4 \times 10^{-9}\right)$ & $0.28794741\left(6.7 \times 10^{-9}\right)$ \\
\hline
\end{tabular}




\begin{tabular}{|c|c|c|c|}
\hline $\mathrm{AL}^{5}$ & & $0.2735501\left(5.0 \times 10^{-8}\right)$ & $-0.28794741\left(7.0 \times 10^{-10}\right)$ \\
\hline $\mathrm{J}-\mathrm{K}$ & 0.03125 & $0.27355005\left(5.0 \times 10^{-1}\right)$ & $-0.28794742\left(4.0 \times 10^{-1}\right)$ \\
\hline $\mathrm{CH} 4$ & & $0.27355003\left(1.0 \times 10^{-8}\right)$ & $-0.28794740\left(1.0 \times 10^{-10}\right)$ \\
\hline $\mathrm{AL}^{5}$ & & $0.2735501\left(6.0 \times 10^{-8}\right)$ & $-0.28794741\left(1.0 \times 10^{-10}\right)$ \\
\hline AB7 & & $0.27354004\left(4.0 \times 10^{-5}\right)$ & $-0.28796321\left(6.0 \times 10^{-5}\right)$ \\
\hline $\mathrm{F}^{4}$ & & $0.2735503\left(3.0 \times 10^{-1}\right)$ & $-0.2879477\left(3.1 \times 10^{-1}\right)$ \\
\hline NM9 & & $0.27354004\left(7.9 \times 10^{-5}\right)$ & $-0.28794740\left(8.3 \times 10^{-1}\right)$ \\
\hline $\mathrm{F}^{5}$ & & $0.27355003\left(6.4 \times 10^{-9}\right)$ & $0.28794741\left(6.7 \times 10^{-9}\right)$ \\
\hline $\mathrm{AL}^{5}$ & 0.05 & $0.2373550\left(1.1 \times 10^{-1}\right)$ & $-0.2879947\left(1.4 \times 10^{-9}\right)$ \\
\hline AB7 & & $0.27354004\left(4.0 \times 10^{-5}\right)$ & $-0.28796321\left(6.0 \times 10^{-5}\right)$ \\
\hline AF5 & & $0.27354738\left(2.7 \times 10^{-6}\right)$ & $-0.28794461\left(1.4 \times 10^{-8}\right)$ \\
\hline \multicolumn{2}{|c|}{ Exact solution } & 0.27355004 & -0.287947411 \\
\hline
\end{tabular}

\section{As shown in the Table 3.1 above, the proposed method in this paper perform better than existingmethods in terms of accuracy.}

\subsection{CONCLUSION}

The aim of this paper was to develop numerical method which provides solution to initial value problems with stiff differential equations via exponentially fitted integrators.Numerical experiments have been carried out using appropriate step size as required by each problem. Such problems which are stiff require small step size before the solution can be smooth. In general, the results from numerical experiment so presented in this paper, show that the new method perform effectively well when compared similar methods in the literature. Hence the aim and objective of this paper have been achieved.

\subsection{REFERENCES}

[1] Abhulimen, C.E and Okunuga, S.A (2008), "Exponentially fitted second derivative multipstep method for stiff initial value problem for ODE's". Journal of Engineering Science and Applications, 5, 36-47

[2] Abhulimen, C.E and Omeike, G.E. (2011). A sixth-order exponentially fitted scheme for the numerical solution of systems of ordinary differential equations". Journal of Applied Mathematics and Bioinformatics, vol. 1 no 1, 175186

[3] Abhulimen, C.E and Otunta, F.O (2006): "A Sixth Order Multi Derivative Multistep Method for stiff System of ODE”. International Journal of Numerical.Maths. (ITNM) 2(1) 248-268.

[4] Abhulimen,C.E. (2009). "Exponential Fitting Predictor-Corrector formula for stiff systems of Ordinary Differential Equations". International Journal of Computational and Applied Mathematics.ISSN 1819-4966 volume 4 no 2, pp 115-126.

[5] Brown,T.L(1977): "Some Characteristics of implicit Multstep Multi-derivative Integration formulas". SIAM J.Numerical Math. 34(1) 59-84.

[6] Cash, J.R. (1981): "On the Exponential Fitting of Composite Multiderivative Linear Multistep Methods". SIAM J. Numerical Annal 18(5), 808-821.

[7] Dahlquist, G. (1963): "A special Stability Problem for Linear Multistep Method”. BIT 3:27-43

[8] Enright, W.H. (1974): "Second Derivative Multistep Methods for Stiff Ordinary Differential Equations", SIAM J. Numerical Analysis II, 321-331

[9] Enright, W.H. and Pryce,J.O(1983), "Two FORTRAN Packages for Assessing IV Methods". Technical Report No .16/83.Department of ComputerScience,University of Toronto, Canada.

[10] Jackson, L.W. and Kenue, S.K (1974): A Fourth -Order Exponentially Fitted Method, SIAM J. Numerical Annal. II, 965-978

[11] Lambert, J.D. (1973). Numerical Method in Ordinary Differential Equations with Initial Value Problem, John Wiley

[12] Liniger, W. and Willioughby, R.A. (1970): Efficient Integration methods for stiff systems of ordinary differential equations, SIAM J. NumerAnnal, 7, 47-65.

[13] Okunuga, S.A (1994). "Composite Multiderivative Linear Multistep methods for Equations from $r$ Stiff IVPs in ODEs".( Ph.D. Thesis), Dept. of Mathematics, University of Lagos Nigeria.

7161 | P a g e 
[14] Okunuga,S.A(1997). Fourth order composite two step method for stiff problems, Int. J. Comput. Math. 2 39-47.

[15] Otunta,F.Oand Abhulimen, C.E. (2005). "A $4^{\text {th }}$ Order Exponentially Fitted Multiderivative method for Stiff IVPs". Nigerian Association of Mathematical Physics. Vol. 9, pp. 295-306.

[16] Voss, D. (1988). "A fifth order Exponentially Fitted Formula”. SIAM. J Number.Anal.25, No 3. 